\title{
Decoherence-free subspaces and spontaneous emission cancellation
}

\author{
K.-P. Marzlin ${ }^{1}$, R. Karasik ${ }^{2,3,1}$, B. C. Sanders ${ }^{1}$, and K. B. Whaley ${ }^{2,3,4}$ \\ 1 Institute for Quantum Information Science, University of Calgary, Calgary, \\ Alberta T2N 1N4, Canada \\ ${ }^{2}$ Applied Science \& Technology Graduate Group, University of California, Berkeley, \\ California 94720, USA \\ 3 Department of Chemistry, University of California, Berkeley, California 94720, \\ $U S A$ \\ ${ }^{4}$ Pitzer Center for Theoretical Chemistry, University of California, Berkeley, \\ California 94720, USA \\ Version: April 17, 2007
}

\begin{abstract}
We establish the connection between decoherence-free subspaces of quantum information and spontaneous emission cancellation in quantum optics with molecules. Both phenomena rely on the destructive interference of transition amplitudes from two initial states to a common target state. We discuss physical similarities, differences, and limitations of both phenomena.
\end{abstract}

\section{Introduction}

Decoherence-free (or noiseless) subspaces (DFS) [1, 2, 3, 4] provide a tool against the effects of decoherence in quantum computation and have been extensively theoretically investigated $[5,6,7,8,9,10,11,12,13,14,15]$. Compared to quantum error correction, which requires active syndrome measurement and feed forward, DFS passively avoid decoherence processes by exploiting the fact that for systems which possess symmetries quantum states within some subspace of the system Hilbert space $\mathcal{H}_{\mathrm{S}}$ may be completely decoupled from the environment. DFS have been applied in quantum information processing to reduce errors due to decoherence [16]. They can help to reduce the amount of quantum error correction required for a given accuracy and in particular to provide longer quantum memory.

In this paper we establish a connection between certain systems that exhibit DFS, and spontaneous emission cancellation (SEC). The latter has been predicted for specific single-particle quantum systems a decade ago [17]. Both phenomena are based on the destructive interference between transition amplitudes from two (or more) different quantum states that are coupled by the environment to a common final state. We remark, however, that this explanation does not apply to all types of DFS. For instance, the ground state of an atom is immune against decoherence due to spontaneous emission simply because of energy conservation, not because of an interference effect. 


\section{Decoherence-free subspaces}

The concept of DFS can be best explained by considering the coupling of a system $S$ to a Markovian reservoir $R$. The (reduced) density matrix $\rho$ of the system then obeys a master equation of Lindblad type [18]

$$
\dot{\rho}=-i[\hat{H}, \rho]+L_{D}[\rho]
$$

In this equation $\hat{H}$ is the system Hamiltonian, including energy level shifts due to the interaction with the environment. Non-unitary dynamics is incorporated into the decoherence propagator

$$
L_{D}[\rho]=\frac{1}{2} \sum_{k, l=1}^{N_{\mathrm{S}}^{2}-1} a_{k l}\left(\left[\hat{F}_{k}, \rho \hat{F}_{l}^{\dagger}\right]+\left[\hat{F}_{k} \rho, \hat{F}_{l}^{\dagger}\right]\right),
$$

where the matrix $A=\left(a_{k l}\right)$ is positive semidefinite and time-independent, $N_{\mathrm{S}}$ the dimension of the system Hilbert space, and the operators $\left\{\hat{F}_{k}\right\}_{k=0}^{N_{\mathrm{S}}^{2}-1}\left(\hat{F}_{0}=\hat{I}\right)$ form a complete basis for the space of bounded operators defined on $\mathcal{H}_{\mathrm{S}}$.

With this dynamical equation a decoherence-free subspace $\mathcal{H}_{\text {DFS }} \subset \mathcal{H}_{\mathrm{S}}$ can be defined as a subspace whose evolution is strictly unitary. In other words, a state $\rho \in \mathcal{H}_{\text {DFS }} \subset \mathcal{H}_{\mathrm{S}}$ must fulfill $L_{D}[\rho]=0$.

For a weak interaction $\hat{H}_{\text {int }}$ between the system and a Markovian reservoir, for which correlations inside the reservoir decay rapidly, Eq. (2) can be derived from an expression that has the general form (see, e.g., Ref. [19])

$$
L_{D}[\rho]=-\frac{1}{\hbar^{2}} \int_{0}^{\infty} \mathrm{d} t^{\prime}\left\langle\left[\hat{H}_{\mathrm{int}},\left[\hat{H}_{\mathrm{int}}\left(t^{\prime}\right), \rho\right]\right]\right\rangle_{R}
$$

where the mean value is taken with respect to the reservoir only and $\hat{H}_{\text {int }}\left(t^{\prime}\right)$ is taken in the interaction picture. One therefore can also find DFS states by studying the interaction Hamiltonian $[2,3]$. We will follow this approach below.

In many theoretical investigations DFS consist of certain entangled states of two or more identical particles. To illustrate this point we consider two identical two-level systems with basis states $\left|g_{i}\right\rangle,\left|e_{i}\right\rangle(i=1,2)$ coupled to a reservoir of harmonic oscillators with annihilation operators $\hat{a}_{n}$. The interaction Hamiltonian then takes the form

$$
\hat{H}_{\mathrm{int}}=\sum_{n} \hat{a}_{n}\left(g_{1, n}\left|e_{1}\right\rangle\left\langle g_{1}\left|+g_{2, n}\right| e_{2}\right\rangle\left\langle g_{2}\right|\right)+\text { H.c. },
$$

with $g_{i, n}$ the coupling constant between system $i$ and oscillator $n$. For a particular $n$, the system state $|D\rangle \sim g_{2, n}\left|g_{1}\right\rangle \otimes\left|e_{2}\right\rangle-g_{1, n}\left|e_{1}\right\rangle \otimes\left|g_{2}\right\rangle$ is not excited by oscillator $\hat{a}_{n}$ because, for an arbitrary reservoir state $\left|\psi_{\mathrm{R}}\right\rangle$ we have

$$
\begin{aligned}
\hat{H}_{\mathrm{int}}|D\rangle \otimes\left|\psi_{\mathrm{R}}\right\rangle= & \sum_{m}\left(g_{2, n} g_{1, m}-g_{1, n} g_{2, m}\right)\left|e_{1}\right\rangle \otimes\left|e_{2}\right\rangle \otimes \hat{a}_{m}\left|\psi_{\mathrm{R}}\right\rangle \\
& +\left(g_{2, n} g_{2, m}^{*}-g_{1, n} g_{1, m}^{*}\right)\left|g_{1}\right\rangle \otimes\left|g_{2}\right\rangle \otimes \hat{a}_{m}^{\dagger}\left|\psi_{\mathrm{R}}\right\rangle .
\end{aligned}
$$


This is an example where the coupling to a particular reservoir mode vanishes due to destructive interference between two transition amplitudes. In the special case $\left|g_{1, n}\right|=\left|g_{2, n}\right|$ the destructive interference also prevents $|D\rangle$ from decaying into the ground state. If the system-reservoir coupling is more symmetric, this effect can be present for all oscillators: if both two-level systems couple in the same way to the reservoir, $g_{1, n}=g_{2, n} \equiv g_{n} \forall n$, the state $|D\rangle=\sqrt{2}^{-1}\left(\left|g_{1}\right\rangle \otimes\left|e_{2}\right\rangle-\right.$ $\left.\left|e_{1}\right\rangle \otimes\left|g_{2}\right\rangle\right)$ is completely decoupled from the reservoir because the transition amplitudes from states $\left|g_{1}\right\rangle \otimes\left|e_{2}\right\rangle$ and $\left|e_{1}\right\rangle \otimes\left|g_{2}\right\rangle$ to $\left|g_{1}\right\rangle \otimes\left|g_{2}\right\rangle$ (or $\left|e_{1}\right\rangle \otimes\left|e_{2}\right\rangle$ ) interfere destructively for each oscillator in the reservoir, see Fig. 1. Hence, in this example, a DFS state $|D\rangle$ exists if both two-level systems are completely identical, including their couplings to the reservoir.

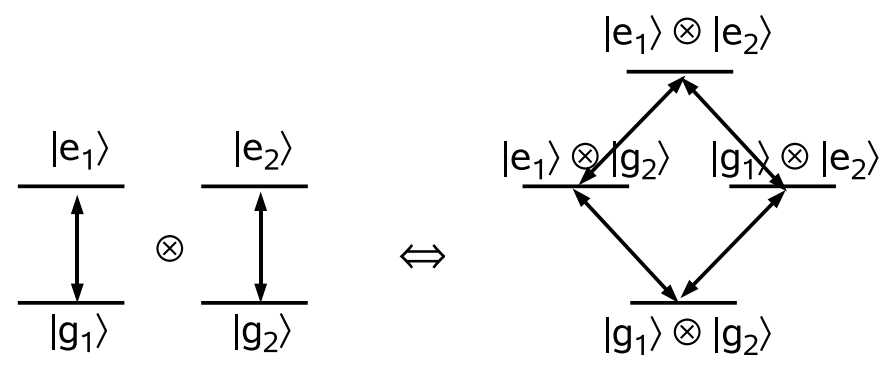

Figure 1: Two identical two-level systems with equal couplings to a reservoir of harmonic oscillators. The entangled state $\left|g_{1}\right\rangle \otimes\left|e_{2}\right\rangle-\left|e_{1}\right\rangle \otimes\left|g_{2}\right\rangle$ is completely decoupled from the reservoir. This is due to destructive interference between the (equal) transition amplitudes from the two degenerate states $\left|g_{1}\right\rangle \otimes\left|e_{2}\right\rangle$ and $\left|e_{1}\right\rangle \otimes\left|g_{2}\right\rangle$ to states with different energy.

This situation can be generalized to $N$ two-level systems, a case that is often studied in DFS theory. For symmetric coupling the Hamiltonian becomes

$$
\begin{aligned}
\hat{H}_{\mathrm{int}} & =\sum_{n} \hat{a}_{n} g_{n}\left(\left|e_{1}\right\rangle\left\langle g_{1}|+\cdots+| e_{N}\right\rangle\left\langle g_{N}\right|\right)+\text { H.c. } \\
& =\sum_{n} \hat{a}_{n} g_{n} \hat{L}^{(+)}+\text {H.c. }
\end{aligned}
$$

with $\hat{L}^{(+)}$a collective excitation operator. As two-level systems are equivalent to spin- $1 / 2$ systems, this operator corresponds to the collective raising operator that appears in angular momentum algebra. A DFS is then composed of states that are eigenstates with eigenvalue zero of this operator $[2,3,20]$ and also eigenstates of the system Hamiltonian. A derivation of the master equation along the lines of Eq. (3) shows that the operator(s) $\hat{F}_{l}$ appearing in Eq. (2) are related to $\hat{L}^{(+)}$. This establishes the connection between the Hamiltonian approach presented here and an approach that is based on a master equation. 


\section{Spontaneous emission cancellation}

In quantum optics the destructive interference between two transition amplitudes plays a vital role in many effects that have been studied during the last two decades. This includes coherent population trapping [21] in which atoms are trapped in a "dark" state, a superposition of two electronic ground states that is not excited by a particular configuration of laser beams. The existence of a dark state and destructive interference is also the key feature for velocityselective coherent population trapping [22] where the velocity dependence of a special dark state is used to cool the atomic center-of-mass motion, electromagnetically induced transparency $[23,21]$ and slow light [24] where an atomic medium becomes transparent and exhibits a very slow group velocity of light because of an atomic dark state, stimulated rapid adiabatic passage [25] to coherently transfer atomic states and Raman adiabatic transfer of optical states [26] to coherently manipulate the quantum state of photons.

All of these phenomena are based on the unitary dynamics of atoms in the presence of classical laser fields. SEC [17] is an interference effect that occurs in the incoherent dynamics of atoms coupled to a reservoir formed by the radiation field. A simple description of SEC can be given for a model of three-level atoms in $\mathrm{V}$ configuration (see Fig. 2). We consider the case of electric dipole coupling

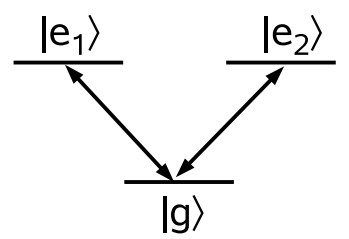

Figure 2: Spontaneous emission cancellation can happen in a particle with three internal levels in $\mathrm{V}$ configuration. If the transition matrix elements are the same destructive interference can generate a stable excited state.

between the particle and the electromagnetic field, which is described by the field operator $\hat{\mathbf{E}}(\mathbf{x})$ at the position $\mathbf{x}$ of the particle. The interaction Hamiltonian then takes the form $\hat{H}_{\text {int }}=-\hat{\mathbf{d}} \cdot \hat{\mathbf{E}}(\mathbf{x})$, where

$$
\hat{\mathbf{d}}=\mathbf{d}_{e_{1}, g}\left|e_{1}\right\rangle\left\langle g\left|+\mathbf{d}_{e_{2}, g}\right| e_{2}\right\rangle\langle g|+\text { H.c. }
$$

is the electric dipole operator of the particle. Expanding the field operator in plane waves $\hat{a}_{\mathbf{k}, \sigma}$ with polarization $\sigma=1,2$ and wavevector $\mathbf{k}$ we find

$$
\begin{aligned}
\hat{H}_{\mathrm{int}} & =-\sum_{\mathbf{k}, \sigma} N_{\mathbf{k}, \sigma} e^{i \mathbf{k} \cdot \mathbf{x}} \hat{a}_{\mathbf{k}, \sigma} \varepsilon_{\mathbf{k}, \sigma} \cdot\left(\mathbf{d}_{e_{1}, g}\left|e_{1}\right\rangle\left\langle g\left|+\mathbf{d}_{e_{2}, g}\right| e_{2}\right\rangle\langle g|\right)+\text { H.c. } \\
& =\sum_{\mathbf{k}, \sigma} \hat{a}_{\mathbf{k}, \sigma}\left(g_{1, \mathbf{k}, \sigma}\left|e_{1}\right\rangle\left\langle g\left|+g_{2, \mathbf{k}, \sigma}\right| e_{2}\right\rangle\langle g|\right)+\text { H.c. }
\end{aligned}
$$

with $g_{i, \mathbf{k}, \sigma}$ the coupling coefficients. Comparing Eq. (8) and Eq. (4) we see that the particular superposition $|D\rangle=\sqrt{2}^{-1}\left(\left|e_{1}\right\rangle-\left|e_{2}\right\rangle\right)$ of excited states is not 
coupled to the ground state $|g\rangle$ if the coupling coefficients do not depend on $i$. This is the case if the dipole moments are equal, $\mathbf{d}_{e_{1}, g}=\mathbf{d}_{e_{2}, g}$, and indicates the appearance of SEC. The destructive interference does not only suppress spontaneous emission but also suppresses excitation by thermal radiation and due to acceleration of the particle (Unruh effect) [19]. The similarity to DFS is now obvious. An intuitive explanation of this similarity can be given by comparing Fig. 2 to the right-hand side of Fig. 1. Concentrating on de-excitation from the middle states we can omit the doubly excited state $\left|e_{1}\right\rangle \otimes\left|e_{2}\right\rangle$ and obtain the same diagram as in Fig. 2. The fact that the two particles are identical guarantees in the case of DFS that the transition matrix elements are equal.

\section{Discussion}

The considerations above imply that one may consider these examples of SEC and DFS as one-particle and multi-particle realizations of the same effect. It remains to discuss how easy it is to realize and utilize these effects. For SEC, selection rules for atomic angular momentum eigenstates forbid that the dipole matrix elements $\mathbf{d}_{e_{1}, g}$ and $\mathbf{d}_{e_{2}, g}$ can be equal. Thus, to realize SEC one has to use molecules. A corresponding experiment has been performed [27] but its results have been questioned [28]. DFS have already been realized for decoherence due to magnetic field fluctuations [11]. However, the construction of multi-particle DFS to suppress spontaneous emission is challenged by our recent result that, for a general system coupled to a homogeneous Markovian reservoir, complete suppression is only possible if the particles are co-located [29]. Because in reality particles are strongly interacting or form molecules at short distances, a true DFS to suppress spontaneous decay does not appear feasible.

Acknowledgments: this project has been funded by iCORE, NSERC, CIFAR, NSF, DARPA and ONR.

\section{References}

[1] L.-M. Duan and G.-C. Guo, Phys. Rev. A 57, 737 (1998).

[2] P. Zanardi and M. Rasetti, Phys. Rev. Lett. 79, 3306 (1997).

[3] D. A. Lidar, I. L. Chuang and K. B. Whaley, Phys. Rev. Lett. 81, 2594 (1998).

[4] P. Zanardi, Phys. Rev. A 57, 3276 (1998).

[5] J. Kempe, D. Bacon, D. A. Lidar, and K. B. Whaley. Phys. Rev. A. 63, 042307 (2001).

[6] A. Shabani and D. A. Lidar, Phys. Rev. A 72, 042303 (2005)

[7] D. A. Lidar, D. Bacon, and K. B. Whaley, Phys. Rev. Lett. 82, 4556 (1999). 
[8] L.-A. Wu and D. A. Lidar, Phys. Rev. Lett. 88, 207902 (2002).

[9] P. G. Kwiat, A. J. Berglund, J. B. Altepeter, and A. G. White, Science 290, 498 (2000).

[10] D. Kielpinski, V. Meyer, M. A. Rowe, C. A. Sackett, W. M. Itano, C. Monroe, and D. J. Wineland, Science 291, 1013 (2001).

[11] C. Langer et al., Phys. Rev. Lett. 95, 060502 (2005)

[12] L. Viola, E. M. Fortunato, M.A. Pravia, E. Knill, R. Laflamme, D. G. Gory, Science 293, 2059 (2001).

[13] L. Duan and G. Guo, Phys. Rev. A 58, 3491 (1998).

[14] D. W. Kribs, R. Laflamme, and D. Poulin, Phys. Rev. Lett. 94, 180501 (2005).

[15] M. D. Choi and D. W. Kribs, Phys. Rev. Lett. 96, 050501 (2006).

[16] C. Langer et al., Phys. Rev. Lett., 95, 060502 (2005).

[17] S.-Y. Zhu and M. O. Scully, Phys. Rev. Lett. 76, 388 (1996).

[18] G. Lindblad, Commun. Math. Phys. 48, 119 (1976).

[19] K.-P. Marzlin and J. Audretsch, Phys. Rev. D 57, 1045 (1998).

[20] D. A. Lidar, D. Bacon, J. Kempe, and K. B. Whaley, Phys. Rev. A 61, 052307 (2000).

[21] E. Arimondo, in Progress in Optics XXXV, E. Wolf (Ed.), p. 259, Elsevier (1996).

[22] A. Aspect, E. Arimondo, R. Kaiser, N. Vansteenkiste, and C. CohenTannoudji, Phys. Rev. Lett. 61, 826 (1988).

[23] S. E. Harris,Phys. Today 50, July 1997, p. 36.

[24] L. V. Hau, S. E. Harris, Z. Dutton, and C. H. Behroozi, Nature 397, 594 (1999).

[25] K. Bergmann, H. Theuer, and B. W. Shore, Rev. Mod. Phys. 70, 1003 (1998).

[26] J. Appel, K.-P. Marzlin, and A. I. Lvovsky, Phys. Rev. A 73, 013804 (2006).

[27] H.-R. Xia, C.-Y. Ye, and S. Y. Zhu, Phys. Rev. Lett. 77, 1032 (1996).

[28] L. Li, X. Wang, G. Lazarov J. Yang, J. Qi, and A. M. Lyyra, Phys. Rev. Lett. 84, 4016 (2000).

[29] R. Karasik, K.-P. Marzlin, B. C. Sanders, and K. B. Whaley, quant$\mathrm{ph} / 0702244(2007)$. 